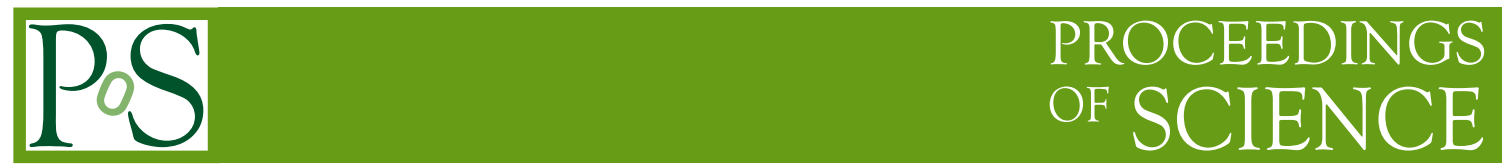

\title{
Four-dimensional formulation of the sector improved residue subtraction
}

\section{David Heymes*}

RWTH Aachen University

E-mail: dheymes@physik.rwth-aachen.de

We report on recent progress we made towards the four-dimensional formulation of the sector improved residue subtraction. We explain how the subtraction scheme STRIPPER, proposed in [1], is generalized to higher multiplicities and therefore furnishes a general framework for the calculation of next-to-next-to-leading order cross sections in perturbative QCD.

Loops and Legs in Quantum Field Theory - LL 2014,

27 April - 2 May 2014

Weimar, Germany

${ }^{*}$ Speaker. 


\section{Introduction}

Upcoming LHC data is expected to reduce the experimental error on several observable cross sections. In order to test the boundaries of the Standard Model, more precise theoretical predictions have to be provided. General tools to calculate not only next-to-leading order (NLO) but also next-to-next-to-leading order (NNLO) cross sections in perturbative QCD are highly demanded. The hadronic cross section factorizes into parton distribution functions and the renormalized partonic cross section

$$
\sigma_{\mathrm{H}_{1} \mathrm{H}_{2}}\left(P_{1}, P_{2}\right)=\int_{0}^{1} \mathrm{~d} x_{1} \mathrm{~d} x_{2} f_{a}\left(x_{1}, \mu_{\mathrm{F}}\right) f_{b}\left(x_{2}, \mu_{\mathrm{F}}\right) \hat{\sigma}_{a b}\left(x_{1} P_{1}, x_{2} P_{2} ; \alpha_{s}\left(\mu_{\mathrm{R}}\right), \mu_{\mathrm{R}}, \mu_{\mathrm{F}}\right),
$$

where the summation over initial state partons $a, b$ (gluons and massless quarks) is understood. For high energy processes the partonic cross section can be calculated in perturbative QCD. Its expansion in $\alpha_{s}$ up to NNLO is

$$
\hat{\sigma}_{a b}=\hat{\sigma}_{a b}^{(0)}+\hat{\sigma}_{a b}^{(1)}+\hat{\sigma}_{a b}^{(2)}+\cdots
$$

where

$$
\begin{aligned}
& \hat{\sigma}_{a b}^{(0)}=\int_{n} \mathrm{~d} \hat{\sigma}_{a b}^{\mathrm{B}}, \\
& \hat{\sigma}_{a b}^{(1)}=\int_{n+1} \mathrm{~d} \hat{\sigma}_{a b}^{\mathrm{R}}+\int_{n} \mathrm{~d} \hat{\sigma}_{a b}^{\mathrm{V}}+\int_{n} \mathrm{~d} \hat{\sigma}_{a b}^{\mathrm{F}}, \\
& \hat{\sigma}_{a b}^{(2)}=\int_{n+2} \mathrm{~d} \hat{\sigma}_{a b}^{\mathrm{RR}}+\int_{n+1} \mathrm{~d} \hat{\sigma}_{a b}^{\mathrm{RV}}+\int_{n} \mathrm{~d} \hat{\sigma}_{a b}^{\mathrm{VV}}+\int_{n+1} \mathrm{~d} \hat{\sigma}_{a b}^{\mathrm{F} 1}+\int_{n} \mathrm{~d} \hat{\sigma}_{a b}^{\mathrm{F} 2} .
\end{aligned}
$$

The multiplicity of the final state ranges between $n$ and $n+2$ particles. Starting at NLO, $\hat{\sigma}_{a b}^{(1)}$, the calculation suffers from soft and collinear singularities that emerge as poles in the dimensional regularization parameter $\varepsilon$, where the dimension is set to $d=4-2 \varepsilon$. Poles appear after integrating out the loop momentum of the virtual cross section $\mathrm{d} \hat{\sigma}_{a b}^{\mathrm{V}}$ or after phase space integration of the additional parton in the final state of the real-radiation cross section $\mathrm{d} \hat{\sigma}_{a b}^{\mathrm{R}}$. After summing the two contributions and the collinear factorization counterterm $\mathrm{d} \hat{\sigma}_{a b}^{\mathrm{F}}$, poles cancel. At NNLO, the cancellation between real and virtual poles continues, but is more involved. It takes place between the double-real cross section $\mathrm{d} \hat{\sigma}_{a b}^{\mathrm{RR}}$, where two additional partons in the final state can become unresolved, the real-virtual cross section $\mathrm{d} \hat{\sigma}_{a b}^{\mathrm{RV}}$, that includes the one-loop matrix element and one additional parton in the final state, the double-virtual cross section $\mathrm{d} \hat{\sigma}_{a b}^{\mathrm{VV}}$, that contains two loop matrix elements, and the collinear factorization counterterms d $\hat{\sigma}_{a b}^{\mathrm{F} 1}$ and $\mathrm{d} \hat{\sigma}_{a b}^{\mathrm{F} 2}$ of different multiplicities.

Subtraction procedures have been introduced to control the divergent structure of higher order calculations. The Catani-Seymour subtraction scheme [2] and the FKS (Frixione-Kunszt-Signer) scheme [3] are commonly used at NLO. At NNLO, Antenna subtraction [4] has been applied to $e^{+} e^{-} \rightarrow 3$ jets [5] and $g g \rightarrow 2$ jets [6]. The $q_{\perp}$-subtraction [7] has been used to calculate Higgs production [7] and $Z$ pair production [8]. In these subtraction schemes poles cancel analytically as can be seen by considering the first contributions of equation (1.5). The double-real cross section is made integrable in four dimensions by imposing single and double unresolved subtraction terms. 
The real-virtual contribution is made integrable by suitable single subtraction terms. Its explicit poles from the virtual amplitude are cancelled analytically by an integrated subtraction term from double-real radiation. Hence, the $n+1$ particle phase space integration is completely finite in four dimensions. The double-virtual contribution has just explicit poles, they are cancelled by all remaining integrated subtraction terms. Again, this part is finite in four dimensions. ${ }^{1}$

The subtraction scheme STRIPPER (SecToR ImProved PhasE space for Real radiation) was originally introduced in [1] and first applied to top-quark pair production at NNLO [9]. Afterwards, it has proven its applicability to other processes of low multiplicity: Higgs + jet [13], charmless bottom quark decay [12], top quark decay [10], single top quark production [11], muon decay [14] and $\mathrm{Z}$ decay [15].

Currently STRIPPER is formulated in the conventional dimensional regularization scheme (CDR), i.e. momenta and spin degrees of freedom of resolved and unresolved particles are treated in $d=4-2 \varepsilon$ dimensions. Unresolved particles are either virtual partons in a loop or real radiated partons that can become soft or collinear. In contrast to analytic subtraction schemes STRIPPER is a numerical approach to calculate cross sections. Therefore, a parametrization of momenta has to be provided for all particles right from the beginning. Using CDR, also unphysical dimensions are parameterized, e.g. for $t \bar{t}$-production already five dimensions had to be parameterized explicitly [9]. Adding an additional particle would increase the dimensionality by one. Furthermore, all matrix elements that are used for subtraction terms have to be provided to higher powers in $\varepsilon$. Unfortunately, available software for leading order matrix elements provide just the four dimensional part. The applicability of STRIPPER in CDR for higher multiplicities is therefore problematic. Hence, it is desireable to reformulate it in the 't Hooft-Veltman regularization scheme (HV), where momenta and spin degrees of freedom of resolved particles are in four dimensions.

The explicit distinction between CDR and HV does not appear in analytic subtraction schemes mentioned above, since all phase space integrations are four dimensional and the difference between CDR and HV starts at order $\varepsilon$.

In this proceedings, we explain the general STRIPPER algorithm used to obtain a Laurent series in $\varepsilon$ for $\hat{\sigma}_{a b}^{\mathrm{RR}}$, where all coefficients can be integrated numerically. Subsequently, we explain corrections that have to be applied in order to reformulate the scheme in HV.

\section{STRIPPER - Outline of the general procedure}

The double-real radiation contribution to the full cross section has the explicit form

$$
\hat{\sigma}_{a b}^{\mathrm{RR}}=\int_{n+2} \mathrm{~d} \hat{\sigma}_{a b}^{\mathrm{RR}}=\frac{1}{2 \hat{s}} \int \mathrm{d} \Phi_{n+2}\left\langle\mathscr{M}_{n+2}^{(0)} \mid \mathscr{M}_{n+2}^{(0)}\right\rangle F_{n+2},
$$

where $\hat{s}$ is the partonic center of mass energy, $\mathrm{d} \Phi_{n+2}$ the $n+2$ particle phase space and $F_{n+2}$ the measurement function which describes the observable under consideration. Matrix elements are described as vectors in color and spin space expanded in $\alpha_{s}$

$$
\left|\mathscr{M}_{n}\right\rangle=\left|\mathscr{M}_{n}^{(0)}\right\rangle+\left|\mathscr{M}_{n}^{(1)}\right\rangle+\left|\mathscr{M}_{n}^{(2)}\right\rangle+\cdots .
$$

\footnotetext{
${ }^{1}$ For simplicity, the collinear counterterms in (1.5) were not mentioned in the discussion.
} 
The squared matrix element appearing in (2.1) is summed over final state color and spin and averaged over initial state color and spin. Momenta and spin degrees of freedom of particles are treated in $d=4-2 \varepsilon$ dimensions. The integral over the phase space will generate poles in $\varepsilon$ as one or two partons become unresolved. The following algorithm provides a systematic approach to calculate (2.1) as a Laurent series in $\varepsilon$ where all integrals can be integrated numerically.

The first step is to introduce selector functions in order to split the phase space into doublecollinear and triple-collinear sectors

$$
1=\sum S_{\mathrm{TC}}+\sum S_{\mathrm{DC}}
$$

In a triple-collinear sector, $S_{\mathrm{TC}}$, singularities are generated as three specific partons become collinear to each other and/or two of them soft. In a double-collinear sector, $S_{\mathrm{DC}}$, singularities are generated as two specific pairs of partons become collinear and/or two of them soft. All remaining soft or collinear limits are regulated by the selector function, which in general depends on energies of final state partons and angles between all partons.

Selector functions are also used to generate subtraction terms at NLO and for the real-virtual cross section at NNLO. In these cases, it coincides with the FKS subtraction method.

The second step is to parameterize the collinear particles in each sector separately using energies and angles. A triple collinear sector, where the three particles are in the final state, is taken as an example: The reference momentum that points towards the triple-collinear direction is denoted by $r^{\mu}$. The momenta of the unresolved partons are denoted by $u_{1}^{\mu}$ and $u_{2}^{\mu}$. Each momentum is parameterized by its energy and a $(d-1)$-dimensional unit vector

$$
r^{\mu} \equiv r^{0}\left(\begin{array}{c}
1 \\
\hat{\boldsymbol{q}}_{1}
\end{array}\right), \quad u_{1}^{\mu} \equiv u_{1}^{0}\left(\begin{array}{c}
1 \\
\hat{\boldsymbol{u}}_{\mathbf{1}}
\end{array}\right), \quad u_{2}^{\mu} \equiv u_{2}^{0}\left(\begin{array}{c}
1 \\
\hat{\boldsymbol{u}}_{2}
\end{array}\right) .
$$

The unresolved particles' energies are rescaled by their maximal value $E$, which depends on the considered process, $u_{i}^{0}=\hat{\xi}_{i} E$, for $i=1,2$. The soft limit is approached as $\hat{\xi}_{1} \rightarrow 0$ and/or $\hat{\xi}_{2} \rightarrow 0$. The unit vectors are defined by

$$
\begin{aligned}
\hat{\boldsymbol{r}} & \equiv \hat{\boldsymbol{n}}^{(d-1)}\left(\alpha_{1}, \alpha_{2}, \ldots\right) \\
\hat{\boldsymbol{u}}_{1} & \equiv \boldsymbol{R}_{1}^{(d-1)}\left(\alpha_{1}, \alpha_{2}, \ldots\right) \hat{\boldsymbol{n}}^{(d-1)}\left(\theta_{1}, \phi_{1}, \rho_{1}, \rho_{2}, \ldots\right), \\
\hat{\boldsymbol{u}}_{2} & \equiv \boldsymbol{R}_{1}^{(d-1)}\left(\alpha_{1}, \alpha_{2}, \ldots\right) \boldsymbol{R}_{2}^{(d-1)}\left(\phi_{1}, \rho_{1}, \rho_{2}, \ldots\right) \hat{\boldsymbol{n}}^{(d-1)}\left(\theta_{2}, \phi_{2}, \sigma_{1}, \sigma_{2}, \ldots\right),
\end{aligned}
$$

where

$$
\hat{\boldsymbol{n}}^{(d-1)}(\alpha, \beta, \ldots)=\left(\begin{array}{c}
\vdots \\
\sin \alpha \sin \beta \ldots \\
\sin \alpha \cos \beta \\
\cos \alpha
\end{array}\right)
$$

$\boldsymbol{R}_{1}^{(d-1)}$ and $\boldsymbol{R}_{2}^{(d-1)}$ are $(d-1)$-dimensional rotation matrices that are chosen in such a way that the scalar products between the three given momenta take the following form

$\hat{\boldsymbol{u}}_{1} \cdot \hat{\boldsymbol{r}}=\cos \theta_{1}=1-2 \hat{\eta}_{1}, \quad \hat{\boldsymbol{u}}_{2} \cdot \hat{\boldsymbol{r}}=\cos \theta_{2}=1-2 \hat{\eta}_{2}, \quad \hat{\boldsymbol{u}}_{1} \cdot \hat{\boldsymbol{u}}_{2}=\cos \theta_{1} \cos \theta_{2}+\cos \phi_{2} \sin \theta_{1} \sin \theta_{2}$. 


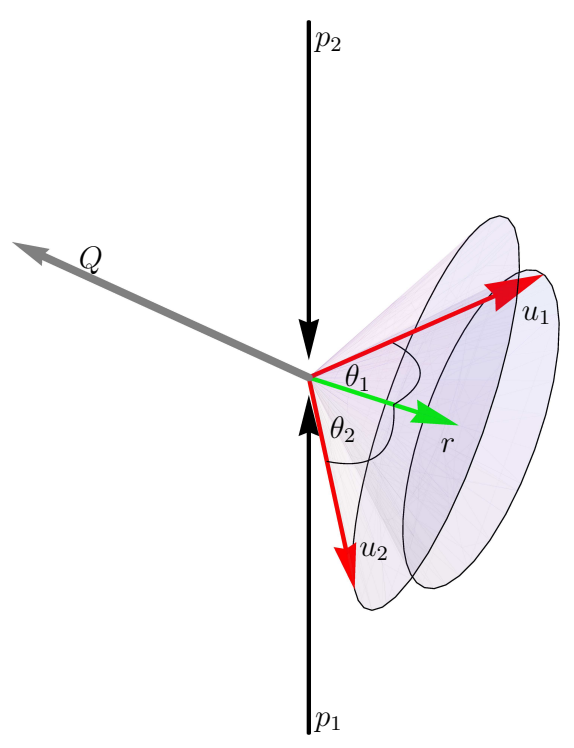

Figure 1: Parametrization of a triple-collinear sector, where the three partons are in the final state. The reference momentum is $r$, the unresolved momenta are denoted by $u_{1}$ and $u_{2}$. The sum of momenta of remaining resolved particles is $Q$.
This parametrization is depicted in figure 1. The limit of $\hat{\eta}_{i}$ at zero indicates the collinear limit of one of the unresolved partons and the reference parton. On the other hand, $\hat{\boldsymbol{u}}_{\mathbf{1}}$ is collinear to $\hat{\boldsymbol{u}}_{2}$ when $\hat{\eta}_{1}=\hat{\eta}_{2}$ and $\phi_{2}=0$. After transforming $\phi_{2}$ non-linearly, it becomes a function of $\hat{\eta}_{1}, \hat{\eta}_{2}$ and a new integration variable $\zeta$

$$
\phi_{2} \rightarrow \phi_{2}\left(\hat{\eta}_{1}, \hat{\eta}_{2}, \zeta\right)
$$

The transformation ensures that $\phi_{2}$ always vanishes as $\hat{\eta}_{1}=\hat{\eta}_{2}$. Using this parametrization just two variables $\hat{\eta}_{1}$ and $\hat{\eta}_{2}$ indicate all possible collinear limits. Furthermore, if a formulation in $\mathrm{HV}$ is found, meaning that all other momenta and the reference momentum are four-dimensional, at most six dimensions are needed to parameterize all possible scalar products that appear.

At this point, four physical variables $\left\{\hat{\eta}_{1}, \hat{\eta}_{2}, \hat{\xi}_{1}, \hat{\xi}_{2}\right\}$ parameterize all possible soft and collinear limits in a given sector. Additional sector decompositions in those variables factorize all possible overlapping singularities that appear at NNLO. For example, in the double-soft limit the phase space integration has the following schematic form

$$
S=\int_{0}^{1} \mathrm{~d} \hat{\xi}_{1} \mathrm{~d} \hat{\xi}_{2} \frac{\hat{\xi}_{1}^{-2 \varepsilon} \hat{\xi}_{2}^{-2 \varepsilon}}{\left(\hat{\xi}_{1}+\hat{\xi}_{2}\right)^{2}}
$$

The integral is singular in the region $\left\{\hat{\xi}_{1}, \hat{\xi}_{2}\right\} \rightarrow\{0,0\}$. Imposing an order on the limits of the soft variables, i.e. splitting the phase space according to

$$
1=\theta\left(\hat{\xi}_{1}-\hat{\xi}_{2}\right)+\theta\left(\hat{\xi}_{2}-\hat{\xi}_{1}\right)
$$

factorizes the singular limit into one variable

$$
S=\int_{0}^{1} \mathrm{~d} \xi_{1} \mathrm{~d} \xi_{2} \frac{\xi_{1}^{-1-4 \varepsilon} \xi_{2}^{-2 \varepsilon}}{\left(1+\xi_{2}\right)^{2}}+(1 \leftrightarrow 2)
$$

where the sector variables $\left\{\xi_{1}, \xi_{2}\right\}$ are functions of the physical variables $\left\{\hat{\xi}_{1}, \hat{\xi}_{2}\right\}$.

The decomposition of the phase space due to soft overlapping singularities is sufficient to factorize all possible limits in a double-collinear sector. In a triple-collinear sector the phase space is split into five additional sectors to factorize collinear and soft-collinear overlapping singularities. 
Finally, the full double-real radiation cross section can be written as a sum over different sectors that are obtained after introducing selector functions (2.3) and applying sector decompositions (2.10)

$$
\hat{\sigma}_{a b}^{\mathrm{RR}}=\sum_{S} \hat{\sigma}_{a b}^{\mathrm{RR}, S}
$$

Each contribution has the following form

$$
\hat{\sigma}_{a b}^{\mathrm{RR}, S}=\int_{0}^{1} \mathrm{~d} \xi_{1} \mathrm{~d} \xi_{2} \mathrm{~d} \eta_{1} \mathrm{~d} \eta_{2} \frac{F_{S}\left(\xi_{1}, \xi_{2}, \eta_{1}, \eta_{2}\right)}{\xi_{1}^{1-b_{1} \varepsilon} \xi_{2}^{1-b_{2} \varepsilon} \eta_{1}^{1-b_{3} \varepsilon} \eta_{2}^{1-b_{4} \varepsilon}},
$$

where all possible singularities are factorized in sector variables. The function $F_{S}\left(\xi_{1}, \xi_{2}, \eta_{1}, \eta_{2}\right)$ is finite as the variables go to zero. This form is appropriate to generate subtraction terms and integrated subtraction terms, i.e. pole terms, using the definition of the plus distribution iteratively for each sector variable

$$
\int_{0}^{1} \mathrm{~d} x \frac{f(x)}{x^{1-b \varepsilon}}=\frac{f(0)}{b \varepsilon}+\int_{0}^{1} \mathrm{~d} x \frac{f(x)-f(0)}{x^{1-b \varepsilon}} .
$$

The result is a Laurent series in $\varepsilon$, where all coefficients can be evaluated numerically. The described procedure is process independent, since it is possible to use the known universal IR-limits of QCD amplitudes for the subtraction and integrated subtraction terms in (2.14). The limit of a vanishing sector variable corresponds to a well defined single or double unresolved limit of the matrix element in (2.1).

\section{Reformulation of STRIPPER in four dimensions}

Treating all particles in $d=4-2 \varepsilon$ dimensions ensures that virtual and real poles arising in the different contributions of (1.5) cancel after all parts are added.

However, the goal is to only treat unresolved particles in $d=4-2 \varepsilon$ dimensions and keep all remaining resolved particles in $d=4$ dimensions. Therefore, it is necessary to identify poles of different contributions in (1.5) that arise from one unresolved particle or from two unresolved particles. The sum of pole terms that arise from one unresolved particle that is proportional to $\left\langle\mathscr{M}_{n+1}^{(0)} \mid \mathscr{M}_{n+1}^{(0)}\right\rangle$ will be called the single-unresolved contribution. The sum of all pole terms that arise from two unresolved particles that is propotional to $\left\langle\mathscr{M}_{n}^{(0)} \mid \mathscr{M}_{n}^{(0)}\right\rangle$ will be called the doubleunresolved contribution. There is one more type of divergent contribution, those proportional to the finite part of the $n$-particle one-loop amplitude $\left\langle\mathscr{M}_{n}^{(0)} \mid \mathscr{F}_{n}^{(1)}\right\rangle$. They arise in $\hat{\sigma}_{a b}^{\mathrm{RV}}, \hat{\sigma}_{a b}^{\mathrm{VV}}$ and $\hat{\sigma}_{a b}^{\mathrm{F} 1}$. It was pointed out that this poles cancel independent of the single- and double-unresolved contributions [16].

A formulation in the 't Hooft-Veltman regularization scheme means that in the single-unresolved contribution, $n+1$ particles are in four dimensions and in the double-unresolved contribution $n$ particles are in four dimensions. This is consistent if it is ensured that these contributions are finite separately, which is the case only after corrections of the integrated subtraction terms in $\hat{\sigma}_{a b}^{\mathrm{RR}}$. How these corrections can be identified will be shortly outlined in section 3.1.

The general procedure to get the transition from CDR to HV is as follows: First, the identified 
corrections for the single-unresolved contribution are added. Then, it is free of poles, i.e. finite in four dimensions. Replacing the measurement function $F_{n+1}$ by

$$
F_{n+1} \cdot \prod_{i=1}^{n} \delta^{(-2 \varepsilon)}\left(q_{i}\right)
$$

restricts $n$ resolved particles of momentum $q_{i}$ to four dimensions. Energy-momentum conservation guarantees that the remaining resolved momentum is also four-dimensional. The error induced by (3.1) is of order $\varepsilon$. In addition, spin-correlated splitting functions are replaced by their azimuthal avaraged counterparts, if they accompany a collinear pole. Finally, momenta and spin degrees of freedom of $n+1$ particles are four-dimensional and only the four-dimensional part of $\left\langle\mathscr{M}_{n+1}^{(0)} \mid \mathscr{M}_{n+1}^{(0)}\right\rangle$ has to be provided.

The corrections to the single-unresolved contribution are subtracted from the double-unresolved contribution and render it finite as well. Then, the same arguments as for the single-unresolved contribution can be applied. The difference is that $n$ particles are resolved. Therefore, the measurement function $F_{n}$ is replaced by

$$
F_{n} \cdot \prod_{i=1}^{n-1} \delta^{(-2 \varepsilon)}\left(q_{i}\right)
$$

After replacing spin-correlated splitting functions by the azimuthal-averaged ones, only the fourdimensional part of $\left\langle\mathscr{M}_{n}^{(0)} \mid \mathscr{M}_{n}^{(0)}\right\rangle$ is needed.

\subsection{Corrections to $\hat{\sigma}_{a b}^{\mathrm{RR}}$ in $\mathbf{H V}$ - An example}

Here we outline how corrections to the integrated subtraction terms in $\hat{\sigma}_{a b}^{\mathrm{RR}}$ can be identified, such that the single-unresolved contribution is finite. First, it is necessary to identify all three parts of the single-unresolved contribution in (1.5). The real-virtual cross section is split in a pure pole contribution and a finite remainder

$$
\begin{aligned}
\int_{n+1} \mathrm{~d} \hat{\sigma}_{a b}^{\mathrm{RV}} & =\frac{1}{2 \hat{s}} \int \mathrm{d} \Phi_{n+1} 2 \operatorname{Re}\left\langle\mathscr{M}_{n+1}^{(0)} \mid \mathscr{M}_{n+1}^{(1)}\right\rangle F_{n+1} \\
& =\frac{1}{2 \hat{s}} \int \mathrm{d} \Phi_{n+1}\left[2 \operatorname{Re}\left\langle\mathscr{M}_{n+1}^{(0)}\left|\boldsymbol{Z}^{(1)}\right| \mathscr{M}_{n+1}^{(0)}\right\rangle+2 \operatorname{Re}\left\langle\mathscr{M}_{n+1}^{(0)} \mid \mathscr{F}_{n+1}^{(1)}\right\rangle\right] F_{n+1},
\end{aligned}
$$

where $\boldsymbol{Z}^{(1)}$ is an operator in color space and contains poles in $\varepsilon$ only. The first term on the r.h.s. of (3.3) is part of the single-unresolved contribution. The collinear factorization counterterm adds

$$
\int_{n+1} \mathrm{~d} \sigma_{a b}^{\mathrm{F}_{2}}\left(p_{1}, p_{2}\right)=\frac{\alpha_{s}}{2 \pi} \frac{1}{\varepsilon} \int_{0}^{1} \mathrm{~d} z\left[P_{c a}^{(0)}(z) \int_{n+1} \mathrm{~d} \sigma_{c b}^{\mathrm{R}}\left(z p_{1}, p_{2}\right)+P_{d b}^{(0)}(z) \int_{n+1} \mathrm{~d} \sigma_{a d}^{\mathrm{R}}\left(p_{1}, z p_{2}\right)\right]
$$

to the single-unresolved contribution, where $P_{a b}^{(0)}(z)$ are the leading order splitting kernels. In the double-real cross section, integrated subtraction terms generated by STRIPPER due to one unresolved parton contribute to the single-unresolved contribution.

If there existed a NLO measurement function in the $n+1$-particle phase space that prevented all particles from becoming soft or collinear, the pole cancellation would be the known NLO pole cancellation between integrated subtraction terms and virtual poles. However, at NNLO one additional particle can become unresolved. Hence, subtraction terms for the soft and collinear limit of 
this particle, which is resolved in this case, have to be taken into account for the single-unresolved contribution. Among those terms the poles do not cancel, because the subtraction terms in $\hat{\sigma}_{a b}^{\mathrm{RR}}$ coincide with the ones in $\hat{\sigma}_{a b}^{\mathrm{RV}}$ and $\hat{\sigma}_{a b}^{\mathrm{F}_{2}}$ only in the limits, but off the limits they do not. This is due to the fact that the subtraction terms in $\hat{\sigma}_{a b}^{\mathrm{RR}}$ are minimal in the sector variables, but not in the physical variables. By comparing terms of similar type, corrections to the double-real subtraction can be identified.

For example, the pole term due to a collinearity in the intial state and a soft subtraction for the resolved particle should cancel between $\hat{\sigma}_{a b}^{\mathrm{RR}}$ and $\hat{\sigma}_{a b}^{\mathrm{F}_{2}}$. Mapping the soft variable of the unresolved parton in the double-real contribution to $z$, the convolution variable in the collinear counterterm, and denoting the soft variable of the resolved particle in both contributions by $\xi$, a direct comparison is possible. The subtraction term is the same in both cases but the integration region is different. As depicted in figure 2 the term in $\hat{\sigma}_{a b}^{\mathrm{RR}}$ is just integrated in the green region, whereas it should also be integrated in the red region to match the contribution in the collinear counterterm. The correction can be added to the subtraction term.

In a similar way all corrections are identified.

\section{Summary and Outlook}

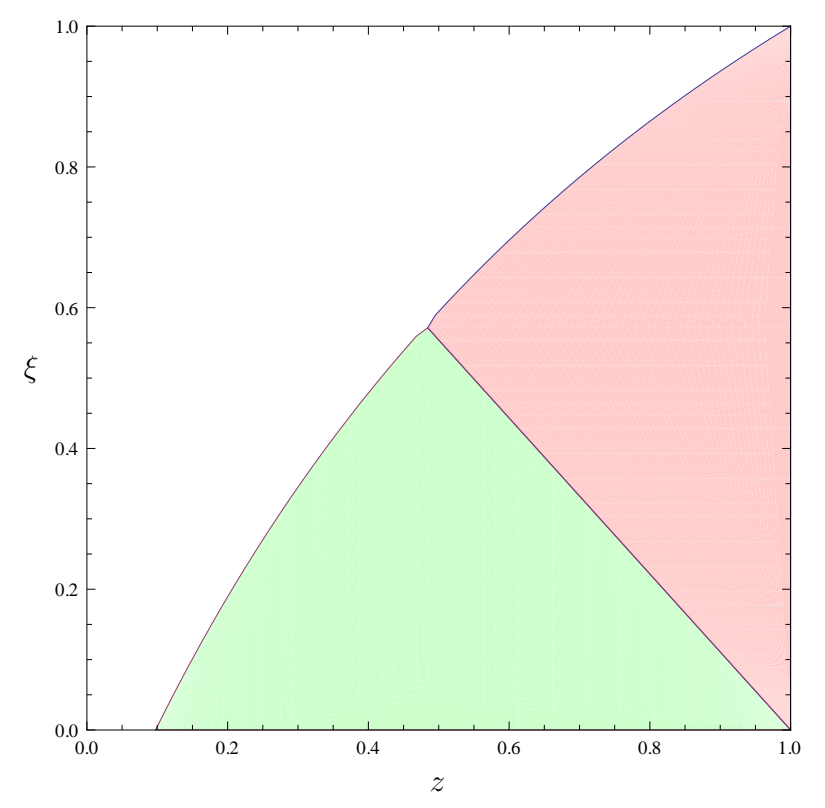

Figure 2: The subtraction term in $\hat{\sigma}_{a b}^{\mathrm{RR}}$ is integrated over the green region of phase space. It should cancel the same subtraction term in $\hat{\sigma}_{a b}^{\mathrm{F}_{2}}$, which is integrated over the red and green region. Therefore, the missing part (red) has to be added to $\hat{\sigma}_{a b}^{\mathrm{RR}}$. Definitions of the variables $\{z, \xi\}$ are given in the text.

In this proceedings, we described the general subtraction scheme STRIPPER and explained how it can be generalized to arbitrary multiplicities by reformulating it in the 't Hooft-Veltman regularization scheme. The main idea was to isolate single-unresolved and double-unresolved contributions and get them finite separately. This was achieved by adding corrections to the integrated subtraction terms in the double-real contribution to the NNLO cross section. Then momenta of resolved particles were put to four dimensions. By replacing spin correlated splitting functions by their azimuthal averaged counterparts, we got rid of spin correlations between four-dimensional matrix elements and $d=4-2 \varepsilon$ dimensional vectors. All matrix elements are just needed in four dimensions. Summing up, the extended formulation of STRIPPER furnishes a general framework to calculate cross sections at NNLO in perturbative QCD. 


\section{Acknowledgments}

This research was supported by the German Research Foundation (DFG) via the Sonderforschungsbereich/Transregio SFB/TR-9 "Computational Particle Physics".

\section{References}

[1] M. Czakon, Phys. Lett. B 693 (2010) 259 [arXiv:1005.0274 [hep-ph]].

[2] S. Catani and M. H. Seymour, Nucl. Phys. B 485 (1997) 291 [Erratum-ibid. B 510 (1998) 503] [hep-ph/9605323].

[3] S. Frixione, Z. Kunszt and A. Signer, Nucl. Phys. B 467 (1996) 399 [hep-ph/9512328].

[4] A. Gehrmann-De Ridder, T. Gehrmann and E. W. N. Glover, JHEP 0509 (2005) 056 [hep-ph/0505111].

[5] A. Gehrmann-De Ridder, T. Gehrmann, E. W. N. Glover and G. Heinrich, JHEP 0711 (2007) 058 [arXiv:0710.0346 [hep-ph]].

[6] J. Currie, A. Gehrmann-De Ridder, E. W. N. Glover and J. Pires, JHEP 1401 (2014) 110 [arXiv:1310.3993 [hep-ph]].

[7] S. Catani and M. Grazzini, Phys. Rev. Lett. 98, 222002 (2007) [hep-ph/0703012].

[8] F. Cascioli, T. Gehrmann, M. Grazzini, S. Kallweit, P. Maierhöfer, A. von Manteuffel, S. Pozzorini and D. Rathlev et al., arXiv:1405.2219 [hep-ph].

[9] M. Czakon, Nucl. Phys. B 849 (2011) 250 [arXiv:1101.0642 [hep-ph]].

[10] M. Brucherseifer, F. Caola and K. Melnikov, JHEP 1304 (2013) 059 [arXiv:1301.7133 [hep-ph]].

[11] M. Brucherseifer, F. Caola and K. Melnikov, arXiv:1404.7116 [hep-ph].

[12] M. Brucherseifer, F. Caola and K. Melnikov, Phys. Lett. B 721 (2013) 107 [arXiv:1302.0444 [hep-ph]].

[13] R. Boughezal, F. Caola, K. Melnikov, F. Petriello and M. Schulze, JHEP 1306 (2013) 072 [arXiv:1302.6216 [hep-ph]].

[14] F. Caola, A. Czarnecki, Y. Liang, K. Melnikov and R. Szafron, arXiv:1403.3386 [hep-ph].

[15] R. Boughezal, K. Melnikov and F. Petriello, Phys. Rev. D 85 (2012) 034025 [arXiv:1111.7041 [hep-ph]].

[16] S. Weinzierl, Phys. Rev. D 84 (2011) 074007 [arXiv:1107.5131 [hep-ph]]. 